\title{
Anaesthetic Considerations in Patients with Cutis Laxa: Our Experience
}

\author{
Dr Sheetal K. ${ }^{1}$, Dr Nandini R. ${ }^{2}$, Dr Chandrika Y. R. ${ }^{3}$ \\ ${ }^{1}$ Assistant Professor, Indira Gandhi Institute of Child Health \\ ${ }^{2}$ Senior Resident, Indira Gandhi Institute of Child Health \\ ${ }^{3} \mathrm{HOD}$, IGICH, Indira Gandhi Institute of Child Health
}

*Corresponding Author: Dr. Sheetal K.; sheetal.kotegar@gmail.com

Received 05 May 2019;

Accepted 18 May 2019;

Published 24 May 2019

\begin{abstract}
Cutis laxa is a rare, inherited or acquired connective tissue disorder. It is characterized by loose, inelastic skin and various systemic involvements. Cutis laxa type III, described as de Barsy syndrome presents with ophthalmic opacification, skeletal involvement, cardiovascular involvement, mental and growth retardation. Intraoperative hyperthermia of the non-malignant variety with tachycardia is seen in $10 \%$ cases of cutis laxa type III. Given the rarity of cutis laxa syndrome, all cases require core and peripheral temperature monitoring.
\end{abstract}

Keywords: cutis laxa, de Barsy syndrome, hyperthermia, temperature monitoring

\section{Introduction}

Cutis laxa is a rare connective tissue disorder that can be inherited or acquired. The patients develop a progeroid appearance and the skin becomes inelastic and hangs loosely in folds. Cutis laxa can also affect connective tissue in other parts of the body including the heart, blood vessels, joints, intestines and lungs.1 In cutis laxa, there is a generalized reduction in the elastic fibers caused by disruption of their normal arrangement.

\section{Case Series:}

\section{Case Report 1}

Case 1 was a 1 year 8 month old male child diagnosed with congenital cutis laxa, planned for bilateral inguinal hernia repair. He presented with loose hanging skin, loud P2, dilated right atrium and ventricle and pulmonary hypertension. This child had a difficult intravenous access and difficult mask ventilation. Induction was done with oxygen, air and sevoflurane. $22 \mathrm{~g}$ cannula was secured in the right EJV following which fentanyl and atracurium was given. Standard ASA monitors with core temperature monitoring were done. Intubation was done with 3.5 $\mathrm{mm}$ uncuffed tube. On direct laryngoscopy, lax vocal cords were seen. Maintenance was with air, oxygen and isoflurane. Caudal analgesia was given with Bupivacaine $0.125 \% 8 \mathrm{ml}$. A rising trend in intraoperative temperature was noted up to $37.5^{\circ}$ celsius. Postoperatively, on extubation, hyperthermia and tachycardia, not responding to intravenous Paracetamol and tepid sponging was observed. The child was shifted to PICU where he developed 1 episode of GTCS and SVT and eventually succumbed.

\section{Case Report 2}

Case 2 was a 3 year old female patient with bilateral DDH planned for spica application. She presented with loose, senile skin and was diagnosed with cutis laxa. The OT was prepared in anticipation for malignant hyperthermia in view of the previous case having a persistently high temperature. Anaesthesia machine was flushed 30 minutes prior to the procedure. Vaporizers were removed. Soda lime canister was changed. Induction was done with Injection Thiopentone, fentanyl, oxygen and propofol. I gel size 2 was inserted. Maintenance was with air, oxygen and propofol infusion. Recovery was uneventful.

\section{Case Report 3}

Case 3 was a 1 month old male child, diagnosed with neonatal cholestasis, planned for an intraoperative cholangiogram. On examination, the neonate had dysmorphic features with loose skin folds and limb deformities. The child presented with an episode of jaundice and seizures on 10th day of life. General anaesthesia, IPPV with TIVA was planned.3.5 uncuffed oral ETT was used. Malignant hyperthermia preparation was done as for the previous case. Recovery was uneventful.

\section{Case Report 4}

Case 4 was a 7 year old female child with left DDH for open reduction and femoral shortening. The child was a known case of cutis laxa diagnosed at birth. She presented with triangular face, protruding mandible, short stature, lax skin, hyperextensibility of joints, frontoparietal eminence. GA and IPPV with TIVA was planned. Epidural catheter was inserted at T12-L1 space. Malignant hyperthermia preparation was done. Recovery was uneventful. 


\section{Case Report 5}

Case 5 was a 2 year old female patient, with bilateral DDH and cutis laxa, planned for closed reduction and cast application.GA with TIVA and malignant hyperthermia preparation was done. Recovery was uneventful.

\section{Case Report 6}

Case 6 was a 1 year 2 month old male child with cutis laxa syndrome and bilateral undescended testis. He was planned for bilateral orchidopexy. OT was prepared with consideration to malignant hyperthermia. GA with TIVA and caudal analgesia was planned. The child presented with global developmental delay, large head, wrinkled skin, low set ears and hypotonia. He had undergone a septic arthrotomy on day 9 of life, details of which were unavailable. An uneventful recovery was observed.

\section{Discussion}

Cutis laxa, a disorder of connective tissue, is characterized by sagging and inelastic skin. Cutis laxa also affects connective tissue in other parts of the body including heart, blood vessels, joints, intestines and lungs.1 Individuals affected with cutis laxa may develop abnormalities including arterial tortuosity, aortic aneurysm, pulmonary arterial hypoplasia, cardiac hypertrophy, pulmonary emphysema, musculoskeletal hypotonia, diverticulae of intestine and bladder. The clinical presentation and mode of inheritance show considerable heterogeneity. Autosomal dominant, autosomal recessive and $\mathrm{X}$ linked recessive forms have been noted in inherited forms. 2 Acquired cutis laxa is generally seen in adults, following an environmental exposure. Cutis laxa is diagnosed based on symptoms, patient history, clinical evaluation and specialized tests. Biopsy of affected skin can reveal characteristic changes in elastic fibres.3 Differential diagnosis of cutis laxa includes Costello syndrome and Ehler Danlos syndrome type III C.4 Ananesthetic concerns include challenging peripheral intravenous access, difficult airway, problems with coagulation and increased risk for peripheral nerve injury with intraoperative positioning. De Barsy syndrome is a rare clinical syndrome characterized by cutis laxa, ophthalmic opacification, skeletal malformation, as well as mental and growth retardation.5 It is transmitted in an autosomal recessive fashion, and is described as cutis laxa type 3 . Previously published reports show associated cardiac anomalies in patients with De Barsy syndrome. Intraoperative hyperthermia has been reported (Aponte, Smith et al 2010) in approximately $10 \%$ cases with temperature exceeding 38 ${ }^{\circ}$ celsius. 6 While there is no evidence of malignant hyperthermia, they were associated with tachycardia. It is possible that these cases represent a form of non- malignant hyperthermia similar to that manifested in patients with other congenital diseases such as Costello syndrome and Osteogenesis imperfecta.7, 8 Hence, close temperature monitoring is recommended intraoperatively. Warner et al conducted a case series on de Barsy syndrome and encountered episodes of intraoperative hyerthermia, difficult airway and difficult intravenous access.9

\section{Conclusion}

Although no conclusive evidence associates cutis laxa with malignant hyperthermia, these cases may represent a form of nonmalignant hyperthermia. Close temperature monitoring is mandatory in these patients until proven otherwise.

\section{References}

[1] Berk DR, Bentley DD, Bayliss SJ, Lind A, Urban Z. Cutis laxa: a review. J Am Acad Dermatol. 2012 May;66(5):842.e1-17. doi:10.1016/j.jaad.2011.01.004. Review.

[2] Marc Zachary Handler, MD(2017) Cutis laxa (Elastolysis)[ online] Emedicine.medscae.com. Available at https://emedicine.medscape.com/article/1074167overview [Accessed 25 March 2019]

[3] Zsolt Urban, Cutis laxa, Rare disese database [online] National organisation for rare disorders. Available at https://rarediseases.org/rare-diseases/cutis-laxa/ [Accessed 25 March 2019].

[4] Davies SJ, Huges HE. Costello syndrome: natural history and differential diagnosis of cutis laxa. J Med Genet 1994;31:486-489

[5] Hugh M Smith, Lindsay Warner Anaesthetic recommendations for patients suffering from De Barsy syndrome. Orphan anasethesia. Available at https://www.orpha.net/data/patho/Ans/en/DeBarsySyndr ome_US_en_ANS_ORPHA2962.pdf [Accessed 25 March 2019]

[6] Aponte EP, Smith HM, Wanekk BJ and Rettke SR. Anaesthesia considerations for ppatients with de Barsy syndrome. J Clin Anaesth 2010; 22:499-504.

[7] Dearlove O, Harper N. Costello syndrome. Paediatr Anaesth 1997;7:476-477.

[8] Furderer S, Stanek A, Karbowski A and Eckardt A. Intraoperative hyperpyrexia in patients with osteogenesis imperfecta. Z Orthop Ihre Grenzgeb 2000;138:136-139.

[9] Warner LL, Olsen DA, Smith HM Paediatr Anaesth. 2018 Jan;28(1):59-62. doi: 10.1111/pan.13283. Epub 2017 Nov 17. 